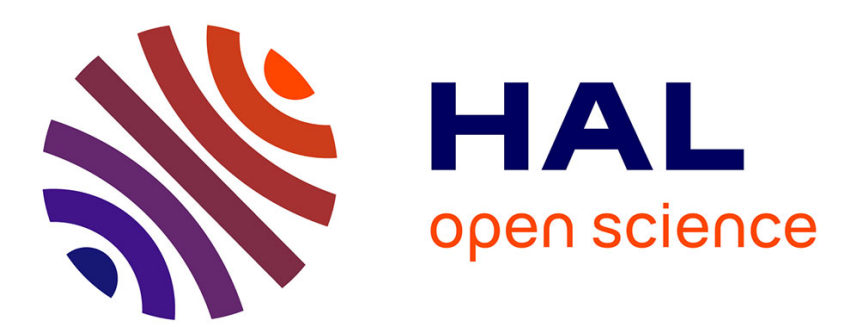

\title{
Caractérisation des formes du bocage à l'aide de la géométrie fractale
}

\author{
B. Roland, Cyril Fleurant
}

\section{To cite this version:}

B. Roland, Cyril Fleurant. Caractérisation des formes du bocage à l'aide de la géométrie fractale. Espace Géographique, 2004, 2, pp.165-174. hal-00735536

\section{HAL Id: hal-00735536 https://hal.science/hal-00735536}

Submitted on 25 Sep 2012

HAL is a multi-disciplinary open access archive for the deposit and dissemination of scientific research documents, whether they are published or not. The documents may come from teaching and research institutions in France or abroad, or from public or private research centers.
L'archive ouverte pluridisciplinaire HAL, est destinée au dépôt et à la diffusion de documents scientifiques de niveau recherche, publiés ou non, émanant des établissements d'enseignement et de recherche français ou étrangers, des laboratoires publics ou privés. 


\title{
Caractérisation des formes du bocage à l'aide de la géométrie fractale Characterization of hedgerow lattice by means of fractal geometry Bernard Roland, Cyril Fleurant
}

\author{
Laboratoire du Paysage, Institut National d'Horticulture, 2, rue Le Nôtre, 49045 Angers cedex
}

RESUME : le paysage bocager a évolué de façon radicale ces cinquante dernières années du fait des développements agricoles. Les formes de la maille bocagère ont des conséquences fondamentales sur les flux du paysage : flux hydrologiques et écologiques plus particulièrement. Aussi nous faisons ici une tentative de caractérisation de la structure du réseau bocager par la géométrie fractale. La méthode du comptage de boîtes nous a permis d'obtenir de bons résultats d'analyse de la maille bocagère par le biais de paramètres de la géométrie fractale (dimension, coupures supérieure et inférieure).

MOTS-CLES : bocage, géométrie fractale, maille bocagère, méthode du comptage de boîtes

ABSTRACT: the bocage landscape has evolved very radically during the last fifty years with the agricultural developement. The shapes of the hedgerow lattice have many consequences for example, in hydrological or ecological functions of this landscape. So we attempt to characterize the network structure by means of fractal geometry. The box-counting method permits to get significantly different results to analyse this structure with fractal parameters (dimension, upper and lower cuts off).

KEY-WORDS : bocage landscape, fractal geometry, hedgerow lattice, box-counting method

\section{Introduction}

Pourquoi analyser le paysage de bocage à l'aide de l'outil fractal ?

Le bocage désigne dans le langage des géographes un paysage agraire d'enclos verdoyants. Il s'oppose au paysage de champs ouverts. Les enclos sont constitués, au sens strict, de haies vives qui entourent complètement les parcelles exploitées. Par extension, des enclos non fermés ou composés de talus, de murets peuvent être qualifiés de bocage (Soltner, 1995). Ses formes sont très diverses et présentent une forte irrégularité ; leur déterminisme est complexe. Les historiens, les géographes, les ethnologues, les agronomes s'y sont intéressés, tentant d'identifier les causes qui ont présidé à ces formes ou d'expliquer leur évolution. Chiva (1991) décrit cette quête et les difficultés rencontrées pour "Comprendre la genèse, dans la longue durée, de ces objets complexes que sont les paysages non urbains, distinguer les paramètres de leur structuration, démêler l'enchevêtrement des facteurs hétérogènes qui les ont façonnés...".

Cet article a été soumis à la revue l'Espace Géographique le 7 octobre 2002.

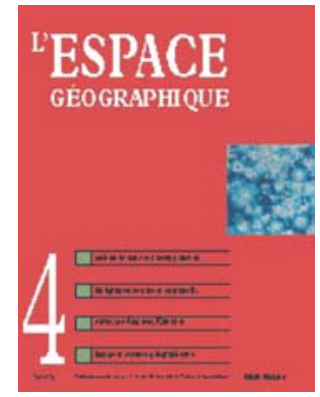


A ce titre, le bocage présente un intérêt particulier, car la structure en réseau du maillage de haies met en relief le tracé du parcellaire et peut faciliter l'analyse de ses formes. Il matérialise l'empreinte des structures agricoles, l'organisation technique et économique d'un pays. En outre, par sa diversité, il marque fortement l'identité d'un territoire. Enfin, l'évolution de ses formes permet une analyse historique. Particulièrement dans les cinquante dernières années, ce paysage a été profondément bouleversé par la modernisation des structures agricoles et la mécanisation. L'augmentation de la productivité s'est souvent accompagnée de l'agrandissement des parcelles :

- la haie devient un obstacle au passage des engins de plus en plus gros,

- les surfaces d'exploitation augmentant, l'entretien des haies devient trop coûteux,

- l'emprise de plusieurs mètres au sol réduit d'autant la surface de production,

- les pouvoirs publics et les politiques de développement rural ont favorisé ces pratiques.

Les différents spécialistes qui se sont intéressés à ce paysage montrent la complexité de ces formes et la décrivent qualitativement sans pouvoir quantifier cette irrégularité. Pourtant l'intérêt de bien connaître les formes qui caractérisent un paysage est évident, notamment dans l'interprétation des flux qui se propagent à différentes échelles.

Plusieurs outils mathématiques sont à la disposition des observateurs pour décrire les formes, notamment la géométrie, qui est fondée sur la caractérisation des propriétés des formes. Cependant, elle atteint rapidement ses limites pour décrire des formes naturelles, dès lors qu'il s'agit de lignes au rayon de courbure infiniment variable par exemple. Les mathématiciens du début du XX siècle parlaient de "courbes pathologiques", de "monstres mathématiques".

Mandelbrot, 1995 cite les courbes de Von Koch, de Peano ou de Sierpinski. Elles résistent à toute tentative de description par les outils mathématiques connus jusqu'alors. La géométrie fractale qu'il invente, dans les années 60, permet de décrire ces courbes dites "non rectifiables ". Il introduit un paramètre caractéristique : leur dimension fractale. "C'est un nombre qui quantifie le degré d'irrégularité et de fragmentation d'un ensemble géométrique ou d'un objet naturel, et qui se réduit, dans le cas des objets de la géométrie usuelle d'Euclide, à leurs dimensions usuelles" (Mandelbrot, 1982; Mandelbrot, 1995)

L'observation montre que les lignes brisées sur un plan ont une dimension fractale comprise entre 1 et 2. Dans l'introduction de son ouvrage "les objets fractals", Mandelbrot (1995) formule de façon intuitive la relation entre la dimension fractale et la forme de l'objet étudié : "Pour caractériser de telles figures, on peut d'abord dire, très grossièrement, qu'une figure dont la dimension se situe entre 1 et 2 doit être plus effilée qu'une surface ordinaire, tout en étant plus massive qu'une ligne ordinaire".

On peut ainsi, par extension, considérer que la dimension d'un objet constitué de lignes qui présentent des directions très variables en couvrant le plan de façon dense, s'approche de 2 et qu'à l'inverse pour un plan peu occupé par des lignes de sinuosité peu contrariée, la dimension est proche de 1. Entre ces deux limites se présente toute la gamme de courbures variablement chahutées et présentant des lacunes plus ou moins grandes.

Chiva (1991) nous parle de "la prise en considération du parcellaire qui permet d'introduire celle des phénomènes d'échelle, d'emboîtement d'échelle et, par là, de discontinuité". Ces éléments descriptifs sont caractéristiques des objets fractals. Il apparaît donc possible de mesurer la dimension fractale d'un maillage bocager.

Dans son ouvrage, Burel (1999) présente plusieurs paramètres caractéristiques des formes. Parmi eux, l'analyse fractale constitue un outil qui décrit l'occupation des sols. 
La dimension fractale d'un paysage bocager, représenté par un réseau maillé en vue aérienne, apparaît donc opportune. La géométrie fractale a été et est toujours utilisée très largement pour décrire et expliquer des objets géologiques (Korvin, 1992) et des phénomènes physiques (Sapoval, 1997; Gouyet, 1992). Mais depuis quelques années, elle est également un outil novateur pour la caractérisation et souvent la compréhension des formes complexes du paysage : les cours d'eau et leur bassin versant (Rodriguez-Iturbe et Rinaldo, 1997; Hauchard et al., 1999), la géomorphologie (Cheng et al., 1999; Nikora et al., 1999; Tyre et al., 1999), l'analyse paysagère ou l'architecture (Duchesne et al., 2001; Bovill, 1995), les réseaux et structures urbaines (Benguigui,1992; Frankhauser, 1994).

L'objet de ce travail est de présenter une analyse de la trame bocagère à l'aide de l'outil fractal afin d'essayer d'en caractériser la forme. Ce travail est l'amorce d'une réflexion plus profonde, et donc de travaux à venir, sur l'influence des structures paysagères (bocages, murettes, haies) et des aménagements anthropiques sur les flux hydriques (inondations, érosions destructives, etc.) et les flux de polluants (transfert des polluants agricoles).

\section{Matériel et méthode}

Nous avons ainsi recherché un site d'étude dans un paysage bocager encore suffisamment dense pour présenter une irrégularité du maillage de haies, avec les caractéristiques suivantes :

- un bassin versant (en vue d'une application ultérieure à l'hydrologie) de 20 à $50 \mathrm{~km}^{2}$,

- un relief marqué,

- un maillage de haies relictuel, ayant évolué au cours des cinquante dernières années, au travers d'épisodes de développement agricole plus ou moins marqués,

- l'existence de prises de vue aériennes du secteur à plusieurs époques durant cette période.

Le département de la Mayenne (France) présente de nombreux sites répondant à ces critères. Celui qui a été retenu se situe en amont du bassin de l'Orthe, sur les communes de Saint-Martin de Connée et Izé (carte de la Figure 1). De plus, il existe dans cette région un programme de réhabilitation et de replantation de haies qui pourrait devenir un terrain d'application des résultats de cette étude théorique.

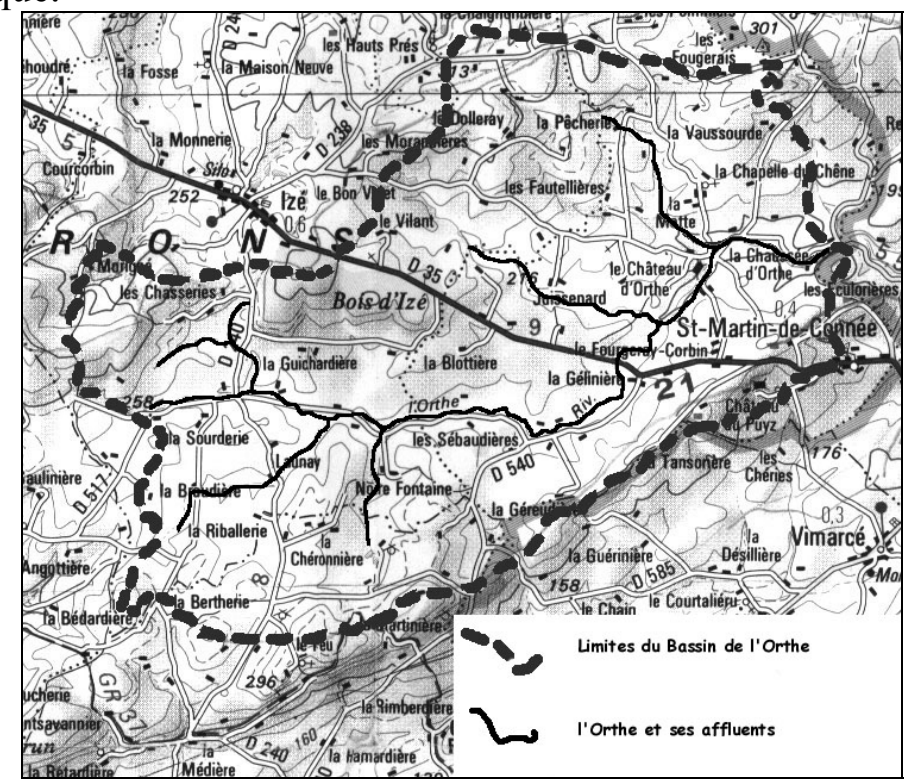

Coordonnées:

$48^{\circ} 10^{\prime} 30^{\prime \prime} \mathrm{N}, 0^{\circ} 12^{\prime} 30^{\prime \prime} \mathrm{W}$ à

$48^{\circ} 14^{\prime} 30^{\prime \prime} \mathrm{N}, 0^{\circ} 20^{\prime} 00^{\prime \prime} \mathrm{W}$

Figure 1 : Carte de situation (extrait IGN) du bassin versant de l'Orthe (échelle Approximative $1 / 107000)$

Par interprétation des photos aériennes (missions IGN 1958 et 1996, échelle : 1/25 000), nous avons reporté le tracé du maillage sur un fond de plan cadastral au $1 / 5000^{\circ}$, afin de respecter les 
limites parcellaires existantes sur lesquelles sont implantées les haies et de tenir compte de la déformation des représentations photographiques.

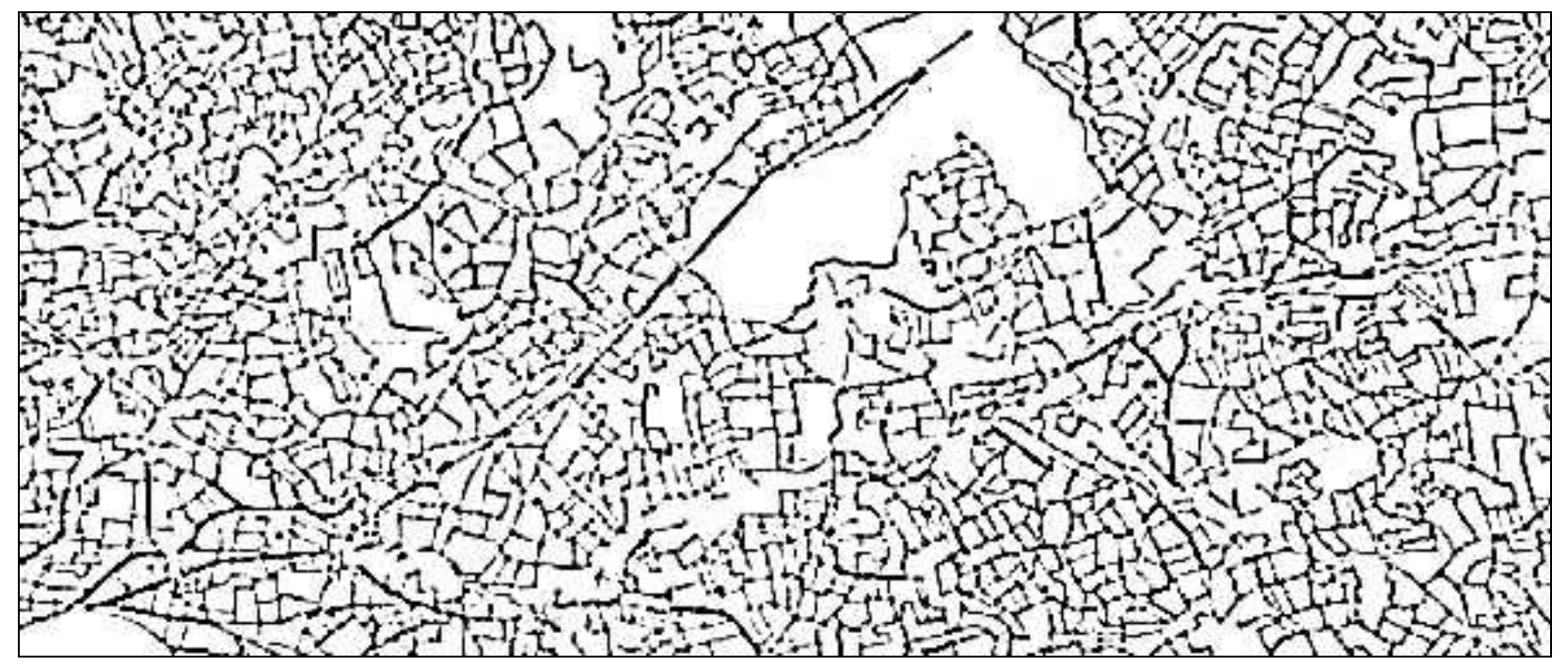

Figure 2 : Report du maillage de haies sur le bassin versant de l'Orthe en 1958 (extrait du plan, échelle approximative : 1/53 000) 


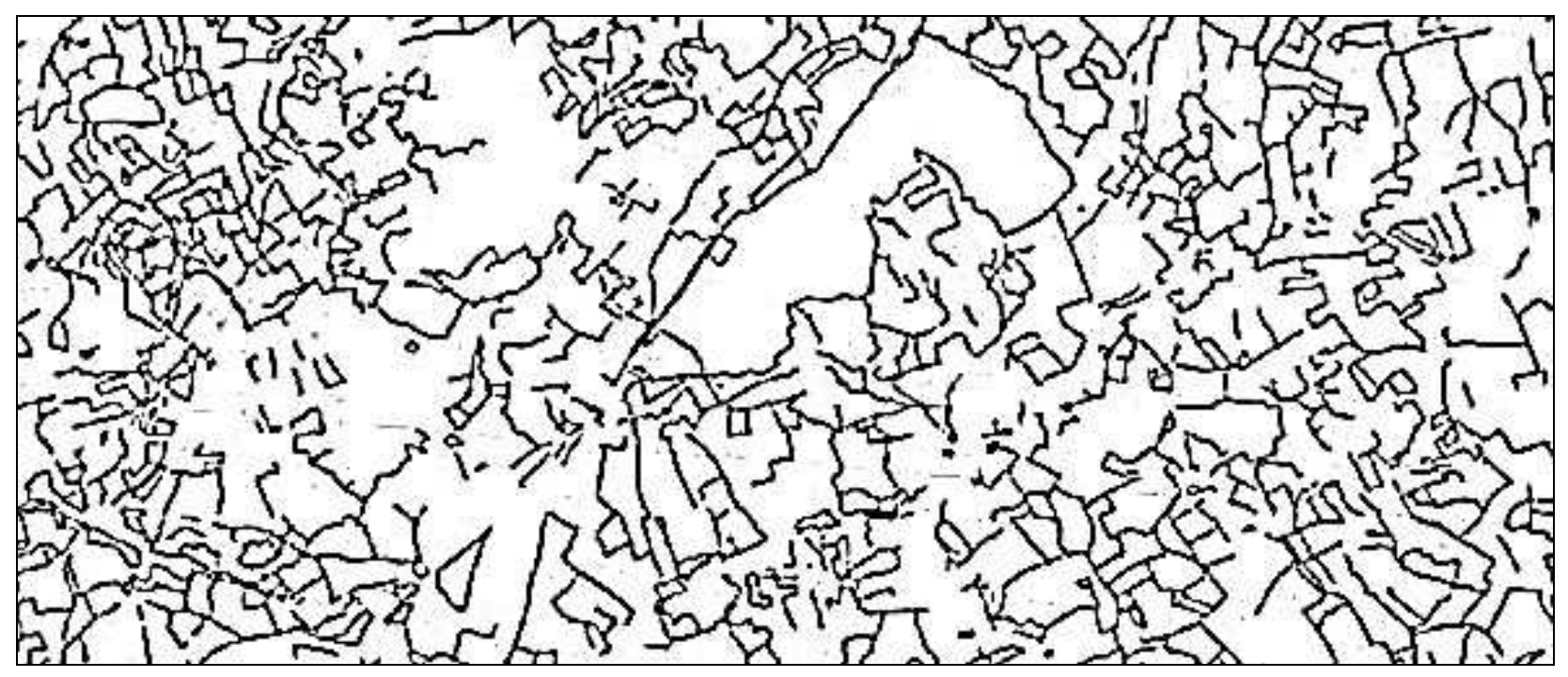

Figure 3 : Report du maillage de haies sur le bassin versant de l'Orthe en 1996 (extrait du plan, échelle approximative 1/53 000)

Nous avons délimité le contour du bassin versant (d'une superficie d'environ $30 \mathrm{~km}^{2}$ ). Afin d'affiner l'analyse de l'évolution et de caractériser des variations dans l'évolution du maillage, à l'intérieur de ce périmètre, nous avons distingué dans les mesures les deux communes du bassin, séparées par une zone "frontière" ; en effet chaque commune possède un historique particulier en matière de foncier :

- St Martin de Connée : Une réorganisation foncière entre 1990 et 1994 ,

- Izé : des échanges amiables échelonnés sur la durée de l'étude.

Pour évaluer la dimension fractale, plusieurs méthodes sont proposées dans la littérature. Nous avons choisi celle que décrit Frankhauser (1994) car il l'applique à la structure urbaine, un milieu fondamentalement anthropique, comme le bocage. Burel (1999) l'utilise également pour caractériser les formes du paysage ou l'occupation du sol.

Il s'agit de la méthode dite du "comptage des boîtes". Frankhauser adapte la formule de Mandelbrot de la façon suivante :

La relation entre le nombre de boîtes pleines et la taille unitaire des boîtes peut s'écrire dans la zone de fractalité :

$$
\mathrm{N} \varepsilon^{\mathrm{D}}=\mathrm{K}
$$


Où

$\mathrm{N}$ : le nombre de boîtes pleines

$\varepsilon$ : la taille unitaire de la boîte

$\mathrm{D}$ : la dimension fractale

$\mathrm{K}$ : une constante

La relation peut encore s'écrire (à une constante près) :

$$
\mathrm{D}=\frac{\log (\mathrm{N})}{\log \left(\frac{1}{\varepsilon}\right)}
$$

La méthode consiste à appliquer successivement sur l'espace que l'on étudie un quadrillage de plus en plus fin et à compter à chaque itération le nombre de cases pleines (i.e. : le nombre de cases qui contiennent au moins une partie de l'objet à mesurer).

On trace le graphe des points $\mathrm{P}_{\mathrm{i}}\left[\log \left(1 / \varepsilon_{\mathrm{i}}\right), \log \left(\mathrm{N}_{\mathrm{i}}\right)\right]$.

La pente de la droite de régression, estimée dans le nuage de points compris dans la zone de fractalité, correspond ainsi à la dimension fractale de l'objet, d'après la relation (2).

Nous avons appliqué la méthode du comptage sur le tracé résultant, en l'adaptant à notre objet. En effet, pour tenir compte de la faible étendue prévisible de la fractalité nous avons débuté le comptage à partir d'une trame constituée de 27 boîtes de $1 \mathrm{~km}$ (longueur terrain) de coté $\left(\varepsilon_{1}=1\right)$. Puis, successivement, ont été appliqués sur la zone d'étude un quadrillage avec des mailles carrées correspondant à une division entière de ce maillage initial, 1/2 km, 1/3 km, 1/4 km, 1/5 $\mathrm{km}, 1 / 6 \mathrm{~km}, 1 / 7 \mathrm{~km}, 1 / 8 \mathrm{~km}, 1 / 9 \mathrm{~km}, 1 / 10 \mathrm{~km}, 1 / 11 \mathrm{~km}, 1 / 12 \mathrm{~km}, 1 / 14 \mathrm{~km}, 1 / 16 \mathrm{~km}, 1 / 24 \mathrm{~km}$, $1 / 32 \mathrm{~km}$ (réalisé à l'aide du logiciel $\operatorname{AUTOCAD}^{\circledR}$ ). Le périmètre de la région étudiée est donc strictement identique, à chaque étape du comptage.

Cette méthode itérative présente l'avantage, par rapport à la méthode proposée par Franckhauser (1994), pour laquelle $\varepsilon$ est divisé par deux à chaque itération, d'augmenter le nombre de points de mesure et d'affiner le résultat dans le cas d'un objet présentant une faible étendue de fractalité.

Nous avons effectué le comptage de boîtes en appliquant la règle suivante : "une boîte est pleine, si elle contient un tronçon de haie ; inversement, elle est vide si elle n'en contient aucun".

\section{Résultats et discussion}


Les résultats du comptage, sur un graphe $\log \left(\mathrm{N}_{\mathrm{i}}\right)$ en fonction de $\log \left(1 / \varepsilon_{\mathrm{i}}\right)$, est donné sur les figures 4 et 5 .

Figure 4 : Résultat du comptage de boîtes sur le bassin versant de l’Orthe amont, en 1958.
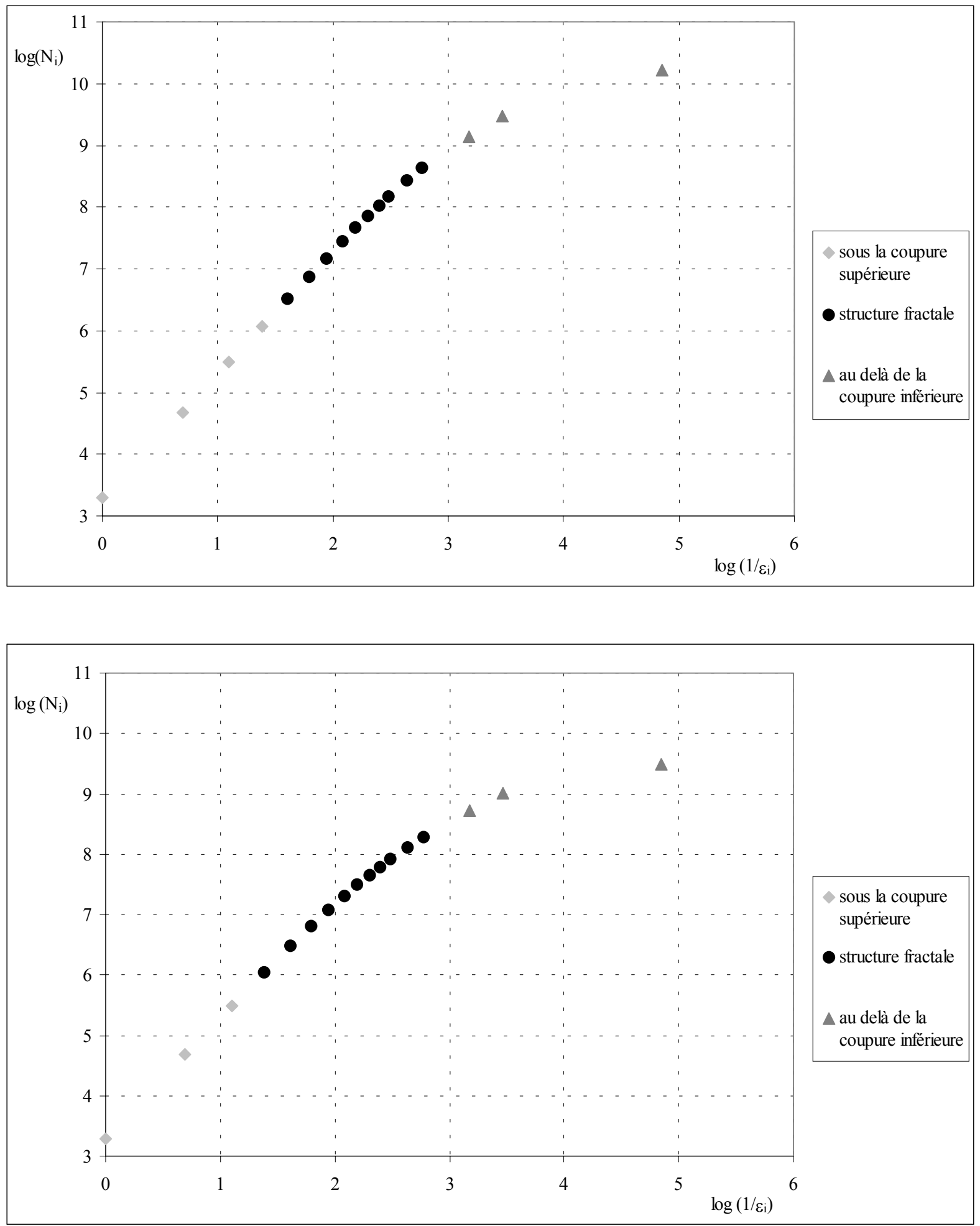

Figure 5 : Résultat du comptage de boites sur le bassin versant de l'Orthe amont, en 1996. 
Les graphes obtenus sont de même type pour l'ensemble des secteurs, et montre que les nuages de points peuvent être séparés en 3 secteurs (Figure 6) :

1. avant la coupure supérieure, la pente de la droite de régression est égale à 2 : toutes les boîtes du quadrillage sont pleines, leur nombre augmente comme le carré de leur taille (figurés par un losange plein sur les graphes)

2. dans la zone de fractalité, la pente est D : la dimension fractale (figurés par un point noir sur les graphes).

3. pour des boîtes de taille inférieure à la coupure inférieure, la pente est 1 car le nombre de boîtes pleines augmente proportionnellement à la taille des boîtes (figurés par un triangle plein sur les graphes).

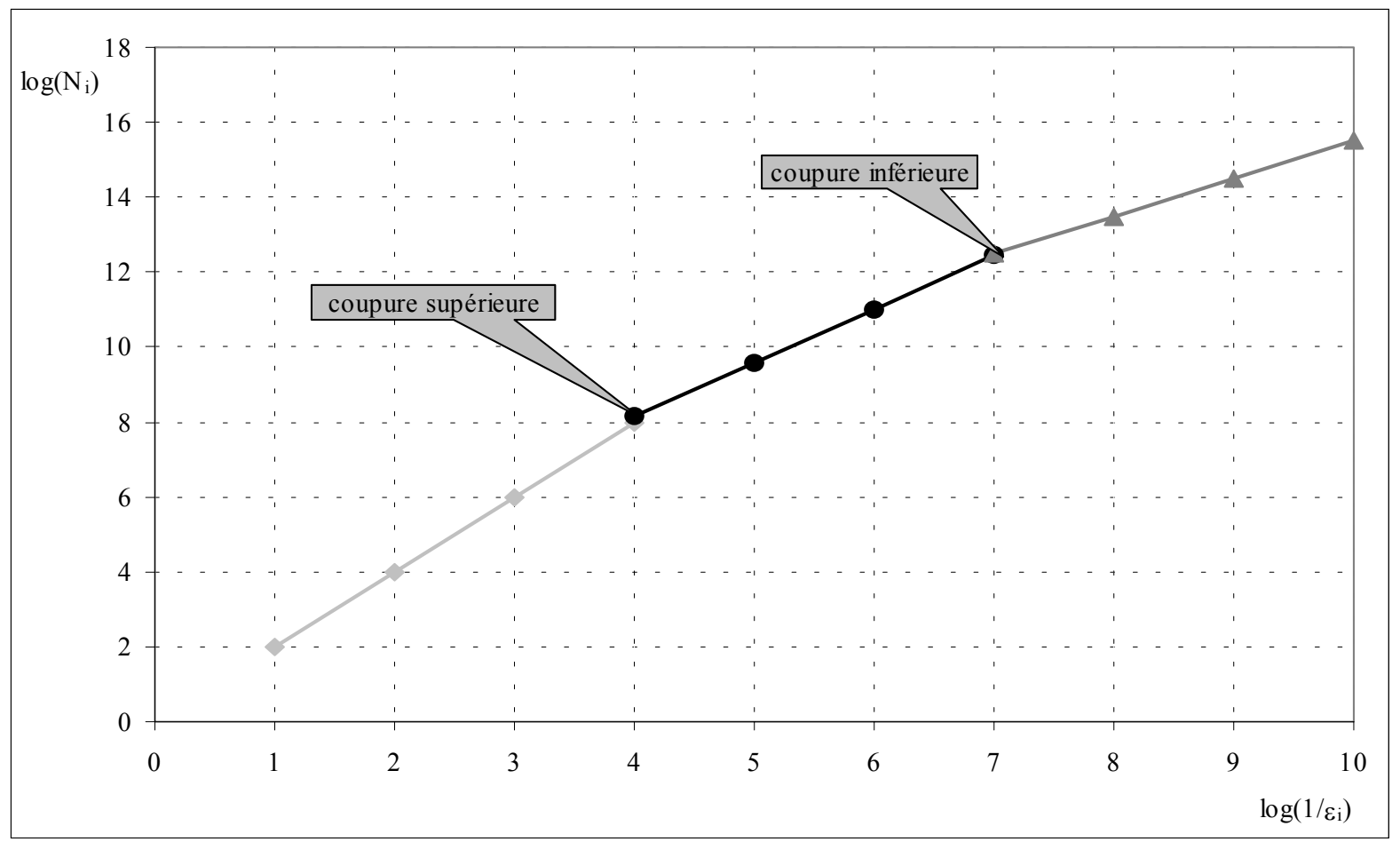

Figure 6 : Courbe théorique présentant la forme générale du nuage de points résultant du comptage de boîtes sur une structure maillée.

La détermination de la dimension fractale revient donc à identifier la zone d'alignement la plus rigoureuse sur les graphes présentés ci-dessus. Celle-ci s'avère délicate car l'étendue de la fractalité est réduite. La détermination des limites de la zone caractérisant le comportement scalant (coupures supérieure et inférieure) apparaît donc essentielle pour identifier cette étendue. Kulatikale et al. (1997) indiquent que la mesure par comptage de boîtes de la plupart des phénomènes naturels révèle le même type de comportement. Il précise que "une des questions les plus critiques lorsque l'on applique la méthode des boites à la mesure d'un objet naturel, est l'identification de la taille de boite à partir de laquelle le nuage de points est aligné dans le graphe en bi-logarithmique. Ce rang est dépendant de l'ordre d'auto-similarité, du facteur de réduction et certainement des coupures inférieure et supérieure caractéristiques".

Il faut ici rappeler la notion de fréquence de coupure et son interprétation intuitive : 
Comme l'indique Sapoval (1997), la détermination des coupures inférieure et supérieure "c'est à dire de la plus petite taille non fractale et de la taille jusqu'à laquelle la géométrie est fractale peut constituer une caractérisation utile".

Dans sa discussion sur la longueur de la côte bretonne, Mandelbrot (1995) définit les notions de coupures externe et interne qui limitent l'étendue du comportement scalant d'un objet fractal.

"Il est raisonnable de supposer que la côte réelle est assujettie à deux coupures. Sa coupure externe se mesure en dizaines ou en centaines de kilomètres. Pour une côte ne se bouclant pas, cette coupure externe pourrait être la distance entre les deux extrémités. Pour une île, elle pourrait être le diamètre du plus petit cercle qui contient toute la côte. D'autre part, la coupure interne se mesure en centimètres".

Par analogie à cette démonstration, concernant l'étude du parcellaire bocager, on pourrait supposer que la coupure supérieure (ou externe) corresponde à une dimension comparable aux plus grands espaces ouverts dans le paysage bocager et que la coupure inférieure (ou interne) soit de l'ordre de la taille des plus petites parcelles entourées de haies sur le parcellaire étudié.

$\mathrm{Si}$ cette hypothèse s'avère exacte, la détermination des coupures supérieure et inférieure permettrait de caractériser de façon quantitative la structure d'un maillage bocager.

L'observation des résultats du comptage nous permet de déterminer la coupure supérieure, car elle correspond à la taille des boîtes pour laquelle il apparaît des boîtes vides dans le périmètre de l'étude. Les résultats de ces coupures sont donnés dans le tableau 1.

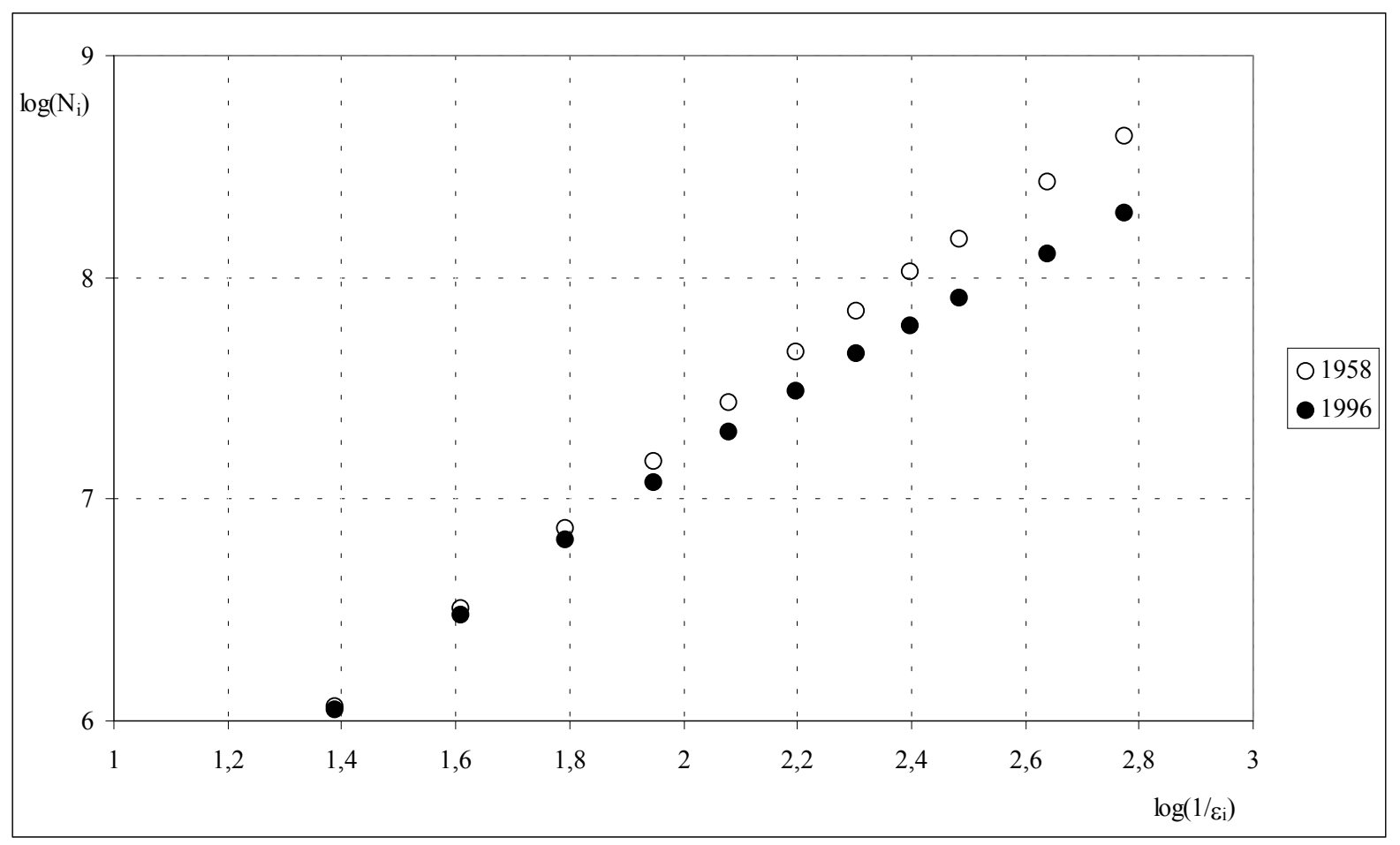

Figure 7 : Extrait des nuages de points sur la zone de fractalité pour les deux dates. 


\begin{tabular}{|c|c|c|c|c|c|c|}
\hline \multirow{2}{*}{ d'observation } & \multicolumn{3}{|c|}{ supérieure } & \multicolumn{3}{c|}{ inférieure } \\
\cline { 2 - 7 } & Izé & St Martin & bv entier & Izé & St Martin & bv entier \\
\hline 1958 & $200 \mathrm{~m}$ & $140 \mathrm{~m}$ & $200 \mathrm{~m}$ & $40 \mathrm{~m}$ & $40 \mathrm{~m}$ & $40 \mathrm{~m}$ \\
\hline 1996 & $250 \mathrm{~m}$ & $200 \mathrm{~m}$ & $250 \mathrm{~m}$ & $70 \mathrm{~m}$ & $60 \mathrm{~m}$ & $70 \mathrm{~m}$ \\
\hline
\end{tabular}

Tableau 1 : relevé des coupures supérieure et inférieure pour les deux dates d'observation et pour les deux communes du bassin versant (bv).

Sur le nuage de points compris entre les deux coupures, on calcule la droite de régression et son critère de dispersion (coefficient $\mathrm{R}^{2}$ ) comme le montre les figures 8 et 9.

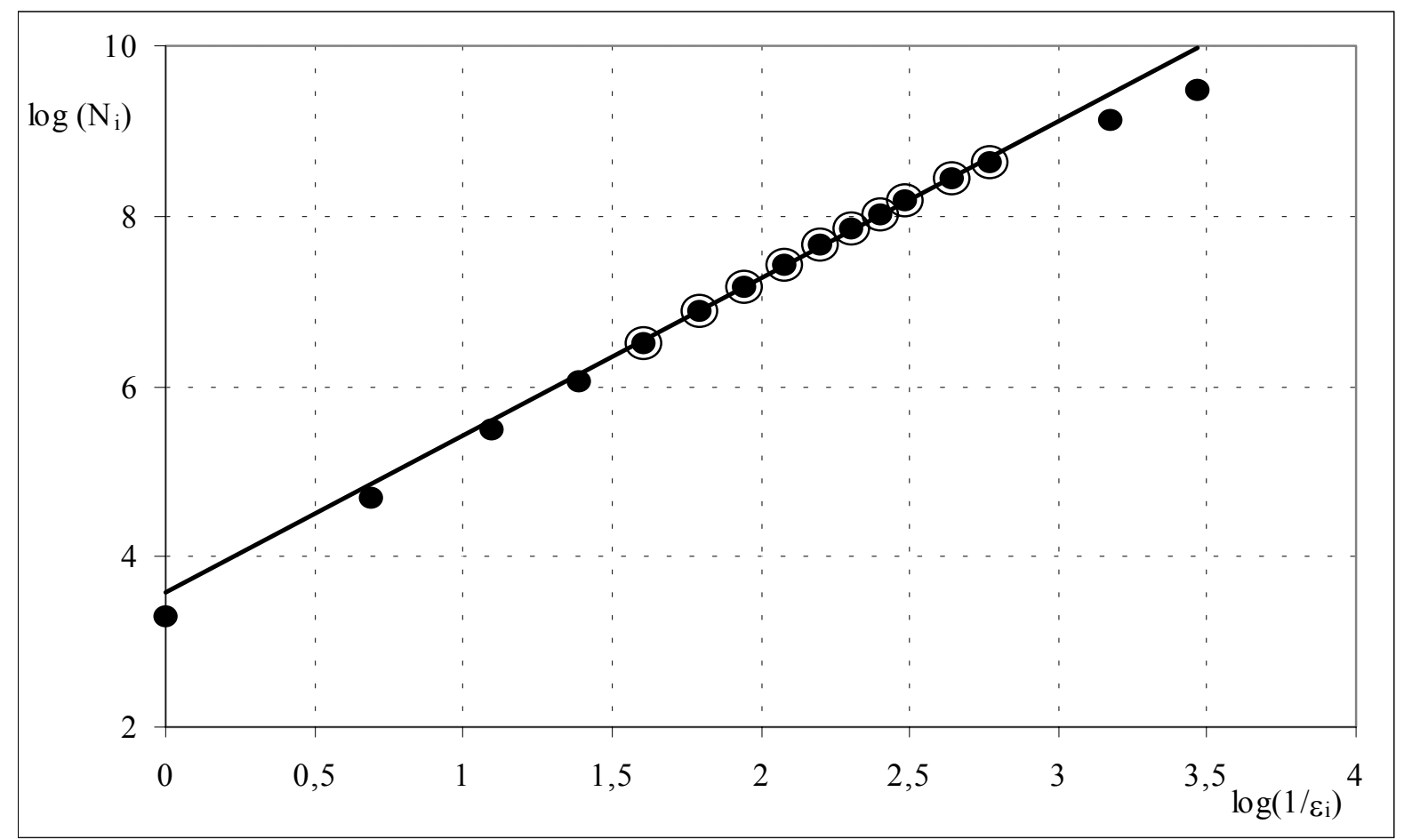

Fig. 8 : Représentation de la droite de régression du nuage de points résultant du comptage de boîtes sur le bassin de l'Orthe en 1958. 


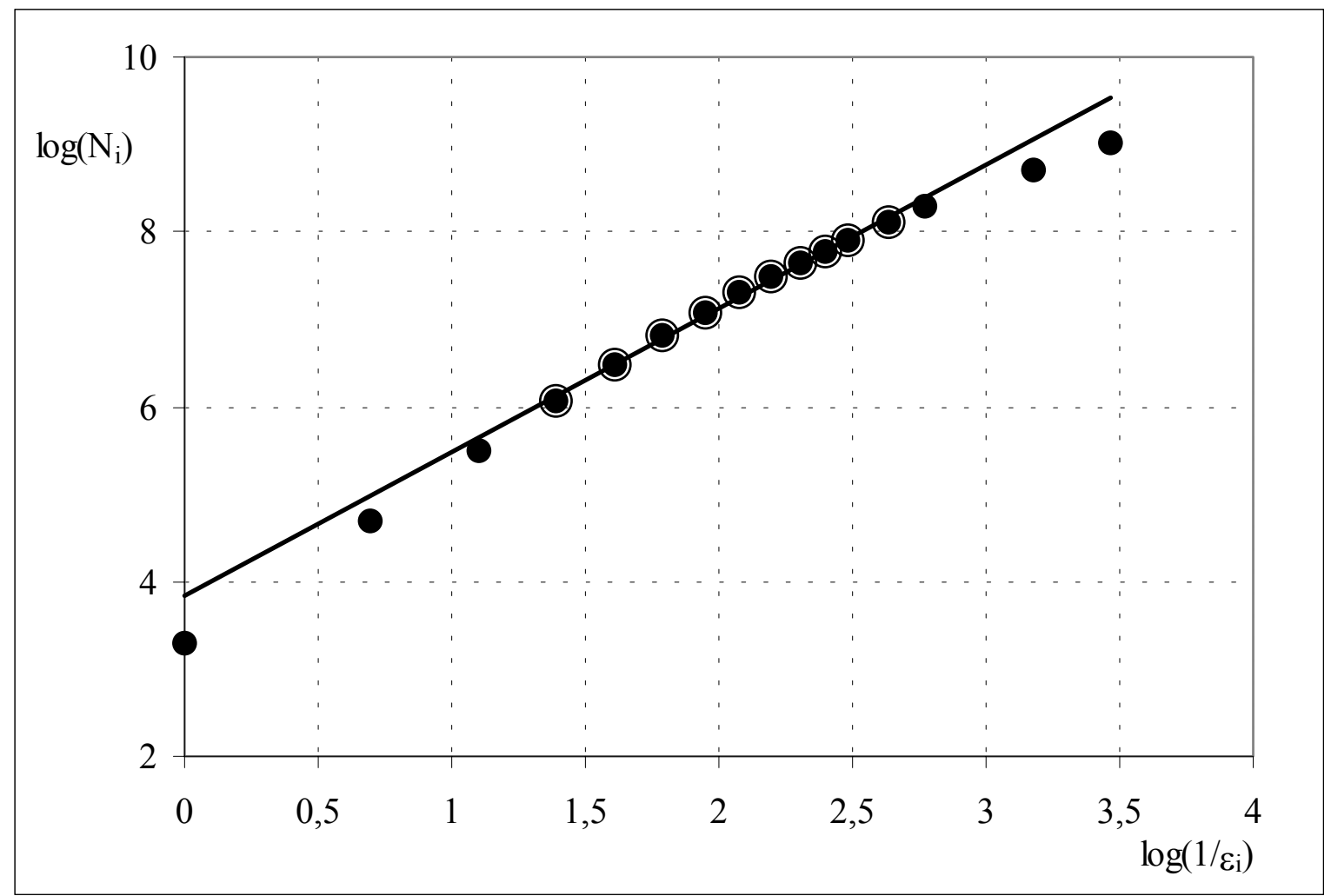

Fig. 9 : Représentation de la droite de régression du nuage de points résultant du comptage de boîtes sur le bassin de l'Orthe en 1996.

Nous avons procédé de la même façon pour les communes de Izé et Saint-Martin de Connée. Les résultats de l'observation sont indiqués dans le tableau 2.

\begin{tabular}{|c|c|c|c|}
\hline $\begin{array}{c}\text { Année } \\
\text { d'observation }\end{array}$ & Izé & $\begin{array}{c}\text { St Martin } \\
\text { de Connée }\end{array}$ & $\begin{array}{c}\text { bassin versant } \\
\text { entier }\end{array}$ \\
\hline 1958 & 1,82 & 1,81 & 1,84 \\
\hline 1996 & 1,69 & 1,57 & 1,64 \\
\hline
\end{tabular}

Tableau 2 : résultat du calcul des dimensions fractales pour les deux communes du bassin versant.

Les coefficients $\mathrm{R}^{2}$ des droites de régression sont tous supérieurs à la valeur 0,99 . Ces résultats montrent un alignement très satisfaisant des points dans la zone de fractalité, vérifiant ainsi notre hypothèse sur la détermination des coupures supérieure et inférieure du maillage.

Les résultats nous permettent également de vérifier la pertinence de la mesure et de valider une de nos hypothèses. On obtient en effet un résultat significativement différent, en 1996, entre la commune de Izé et la commune de Saint-Martin, caractérisant l'historique des échanges parcellaires particulier de chacune des deux communes.

L'analyse fractale révèle sur cet exemple des différences significatives qui permettent de caractériser la structure du réseau maillé. En 1958, le réseau est constitué principalement de petites parcelles, fermées et connectées entre elles. En 1996, le réseau est ouvert et parfois très déstructuré. La dimension fractale peut également être interprétée comme un indicateur de la lacunarité du réseau. Cheng (1999) propose des outils qui permettent de la caractériser. 
La piste ouverte ici semble donc intéressante à explorer afin de corréler la valeur de la dimension fractale avec d'autres paramètres caractéristiques de la structure du maillage bocager.

\section{Perspectives et conclusion}

Ces premiers résultats permettent de montrer qu'il est possible de caractériser les formes du bocage à l'aide de la géométrie fractale. La déstructuration du maillage bocager se traduit par une diminution de la dimension fractale. Nous avons également montré que même si l'irrégularité du maillage est importante, l'étendue de la fractalité est réduite ; ceci nous conduit à manipuler cet outil avec précaution. Toutefois, nous pensons que la dimension fractale de la trame bocagère apporte un élément de connaissance nouveau.

En outre, l'analyse fractale permet de déterminer une fréquence de coupure supérieure et une fréquence de coupure inférieure, caractéristiques d'une trame bocagère. Dans notre étude, elles se distinguent dans tous les cas entre un maillage dense et bien connecté, avant aménagement foncier, et un réseau déstructuré qui résulte des agrandissements de parcelles agricoles. La mesure de ces grandeurs apporte donc également un élément de caractérisation de la structure maillée du bocage.

Ces informations pourraient être utilisées au même titre que d'autres paramètres, tels que la densité de haies, la connectivité du réseau dans des modèles de fonctionnement de ce paysage : physique (hydrologie, ruissellement et érosion) ; chimique (diffusion des micro-polluants) ou biologiques (corridors écologiques...).

L'hydrologie, en particulier, paraît être une voie d'application possible. Le phénomène de percolation est modélisé à l'échelle microscopique à l'aide de la géométrie fractale. Schroeder (1991) s'intéresse à la notion de seuil de percolation sur un réseau à partir de la probabilité d'occupation des mailles d'une trame carrée. Nous envisageons d'appliquer ces travaux à la trame macroscopique du bocage.

Cette première analyse sur un territoire test laisse déjà entrevoir des perspectives de développement et d'application intéressantes. Les résultats obtenus pourraient également contribuer à quantifier des indicateurs utilisés dans l'analyse paysagère. La dimension fractale et ses coupures supérieure et inférieure pourraient à l'avenir être utilisés comme indicateur dans l'analyse paysagère. On pourra aussi proposer de les corréler à certains codes visuels utilisés par les paysagistes tels que la profondeur, la transparence, l'ouverture d'un paysage. La lacunarité nous semble un bon indice pour mesurer ces caractéristiques.

\section{Remerciements :}

À Gérard Clouet (Chambre d'Agriculture de la Mayenne) pour sa collaboration dans le choix du site d'étude grâce à sa parfaite connaissance du terrain et des milieux bocagers. À Jean Duchesne (Professeur, responsable du Laboratoire du Paysage de 1'INH - Institut National d'Horticulture Angers, France) pour la relecture de ce texte.

\section{Bibliographie :}

Benguigui, L. (1992), "Some speculation on fractals and railway networks", Physica A, 191, p. 75-78.

Bovill C, (1995), Fractal geometry in architecture and design, Ed., 190 p.

Burel F. et Baudry J. (1999), Ecologie du Paysage, TEC \& DOC, Paris, New-York, London. Cheng Q. (1999), "The gliding box method for multifractal modeling", Computers and Geosciences, 25, p. 1073-1079.

Cheng Y., Lee P. et Lee T., (1999), "Self-similarity dimensions of the Taiwan island landscape", Computers and Geosciences, 25, p. 1043-1050. 
Chiva I. (1991), "Pour une grammaire du paysage agraire", Etudes Rurales

Duchesne J., Tanguy F. et Joliet F., (2001), How does the fractal geometry lead to new identification of the landscape shapes ?, $38^{\text {th }}$ IFLA World Congress 2001, p. 59-69.

Frankhauser P. (1994), La fractalité des structures urbaines, Ed. Anthropos, 291 p.

Gonzato G., (1998), "A practical method implementation of the box counting algorithm", Computers and Geosciences, 24 (1), p. 95-100.

Gouyet J-F., (1992), Physique et structures fractales, Ed. Hermès, 185 p.

Hauchard E., Delahaye D. et Frankhauser P., (1999), "Analyse morphologique de talwegs et comportements scalant. Applications des dimensions non entières à la géographie physique", L'espace Géographique, 3, p. 215-225.

Korvin G., (1992), Fractal models in the earth sciences, Elsevier, 396 p.

Mandelbrot B. (1982), The fractal geometry of nature, Ed. Freeman and Co, 280 p.

Mandelbrot B. (1995), Les Objets Fractals, Ed. Flammarion, 212 p.

Nikora V., Pearson C. and Shankar U., (1999), "Scaling properties in landscape patterns: New Zealand experience", Landcape Ecology, 14, p. 17-33.

Pinnaduwa H., Fielder R. et Panda B., (1997), "Box fractal dimension as a measure of statistical homogeneity of jointed rock masses", Engineering Geology, 48, p. 217-229.

Rodriguez-Iturbe I. et Rinaldo A., (1997), Fractal river basins, Cambridge University Press

Sapoval B. (1997), Universalités et fractales, Ed. Flammarion.

Schroeder M. (1991), Fractals, Chaos, Power laws, Ed. Freeman and Company.

Soltner D. (1995), L'arbre et la haie, Ed. Sciences et Techniques Agricoles.

Stamps A., (2002), "Fractals, skylines, nature and beauty", Landscape and Urban Planning, 913, p. $1-22$.

Tyre A., Possingham H. and Lindenmayer D., (1999), "Modelling dispersal behavior on a fractal landscape", Environmental Modelling and Software, 14, p. 103-113. 